\title{
Cyclotron radiation of semiconductor crystals
}

\author{
G.V. Milenin, V.V. Milenin, R.A. Red'ko \\ V. Lashkaryov Institute of Semiconductor Physics, National Academy of Sciences of Ukraine \\ 45, prospect Nauky, 03680 Kyiv, Ukraine
}

E-mail: milenin.gv@gmail.com

\begin{abstract}
We obtained relations for estimating the power of cyclotron radiation of semiconductor crystals, when the plasma of thermal carriers is not in a state of thermodynamic equilibrium. It has been shown that the radiation power is a power function of the magnetic field induction. The theoretical calculations are in good agreement with the experimental results.
\end{abstract}

Keywords: cyclotron frequency, cyclotron radiation, semiconductor crystal.

doi: https://doi.org/10.15407/spqeo21.01.054

PACS 76.40.+b

Manuscript received 07.02.18; revised version received 22.03.18; accepted for publication 29.03.18; published online 29.03.18.

\section{Introduction}

A number of publications was devoted to the investigation of radiation from semiconductor crystals in magnetic fields. In particular, the radiation of semiconductors in crossed electric and magnetic fields and generation of terahertz pulses with picosecond duration under irradiation of the semiconductor surface in magnetic field by femtosecond laser pulses were analyzed [1-3].

In [4] the results of an experimental study of highfrequency electromagnetic radiation of germanium crystals in a magnetic field in the absence of any additional influences are presented. It is shown that cyclotron radiation is not in a state of thermodynamic equilibrium with a semiconductor crystal.

Cyclotron radiation of semiconductor crystals is of scientific and practical interest. The latter circumstance is due to the possibility of a smooth tuning of the radiation frequency by changing the induction of magnetic field. In addition, and this is most significant, since in semiconductor crystals the effective mass of charge carriers can be tenths and hundredths of the mass of free electron, the frequency of cyclotron radiation in magnetic fields can be in the microwave and terahertz ranges.

To complete the analysis of this radiation, it is necessary to calculate its power. This paper is devoted to theoretical aspects for calculation of the radiation power.
2. Cyclotron radiation power of a non-equilibrium plasma of thermal charge carriers of semiconductor crystals

When a semiconductor crystal is placed in a homogeneous magnetic field with constant induction $B$, conduction electrons, when their effective mass $m_{n}$ is a scalar quantity (isotropic mass), begin to move along spiral trajectories, that is, helical lines, the axis of which coincides with the magnetic field induction line. It means that the particle simultaneously participates in two motions: under the action of the Lorentz force it rotates uniformly at a velocity $v_{\perp}$ along the circle $\left(v_{\perp}\right.$ is the component of the thermal velocity of electron perpendicular to the vector of magnetic induction) and moves translationally in inertia - uniformly and rectilinearly with a constant velocity $v_{\|}$that is a constituent of the thermal velocity of electron, parallel to the vector of magnetic induction.

The angular velocity of rotation of electron is called the cyclotron (Larmor) frequency $\omega_{B}$. For nonrelativistic particles (for electrons in a semiconductor crystal, the thermal velocity is much lower than the speed of light), it is equal [5]

$\omega_{B}=\frac{e B}{m_{n}}$,

where $e$ is the elementary electric charge (charge of electron). 
Since during rotation electron undergoes acceleration, which is constant in its magnitude and directed perpendicularly to the velocity, it is the source of radiation of electromagnetic waves at the frequency $\omega_{B}[5]$.

Further, simplifying the consideration, we analyze radiation of electron moving along a circle. The radiation power of nonrelativistic electron (the total amount of radiated energy per unit time) at the frequency $\omega_{B}$ is equal [5]

$P=\frac{e^{2} \omega_{B}^{2} v_{\perp}^{2}}{6 \pi \varepsilon_{0} \varepsilon c^{3}}$,

where $\varepsilon_{0}$ is the vacuum electric constant, $\varepsilon$ - dielectric constant of the semiconductor crystal.

The radiation power of electromagnetic waves $P_{n}$ by electrons in a unit volume of a semiconductor crystal, assuming that for the entire array of electrons $v_{\perp}$ is the deterministic quantity, has the form

$P_{n}=n P$,

where $n$ is the concentration of free electrons in semiconductor.

However, in a semiconductor crystal, the vectors of velocity of electrons participating in thermal motion have all possible directions with respect to the vector of magnetic induction. In other words, $v_{\perp}^{2}$ is a random variable ranging from 0 to $v_{T}^{2}$, where $v_{T}$ is the mean square thermal velocity of electrons equal $v_{T}=\left(3 k T / m_{n}\right)^{1 / 2}$, where $k$ is the Boltzmann constant, $T$ - absolute temperature. We will assume that this random variable is a subject to a continuous uniform distribution. Then, the probability density of such a random variable has the form [6]

$f\left(v_{\perp}^{2}\right)=\frac{1}{v_{T}^{2}}$.

Consequently, for the radiation power of electromagnetic waves by electrons per unit volume of a semiconductor crystal, we have

$P_{n}=n \int_{0}^{v_{T}^{2}} P f\left(v_{\perp}^{2}\right) d v_{\perp}^{2}$.

Integrating, we obtain

$P_{n}=\frac{n e^{2} \omega_{B}^{2} v_{T}^{2}}{12 \pi \varepsilon_{0} \varepsilon c^{3}}$.

The cyclotron radiation power of a semiconductor crystal is, respectively, equal to

$P_{S}=\frac{n e^{2} \omega_{B}^{2} v_{T}^{2}}{12 \pi \varepsilon_{0} \varepsilon c^{3}} V$.

As it was shown in [4], not the entire semiconductor crystal radiates, but only a certain near-surface region, and the plasma of the outer radiating layer is not in a state of local thermodynamic equilibrium, and the cyclotron radiation of thermal electrons becomes thermodynamically non-equilibrium.
The degree of deviation of the plasma from the state of thermodynamic equilibrium has a limit. The energy of a cyclotron radiation quantum should not exceed the average kinetic energy of the thermal motion of charge carriers

$\hbar \omega_{B \max }=\frac{3}{2} k T$,

where $\hbar$ is the Planck constant devided by $2 \pi$.

At $T=300 \mathrm{~K}$, we have $v_{B \max }=9.38 \cdot 10^{12} \mathrm{~Hz}$ $\left(\omega_{B}=2 \pi v_{B}\right)$.

The latter circumstance, according to (7), limits the power of cyclotron radiation.

When applying (7), it is necessary to have a notion of the thickness of the radiating plasma layer.

Suppose that a semiconductor crystal has the shape of a cylindrical ingot with a base area $S$ and a height $\delta$ equal to the thickness of the radiating layer, and the magnetic field induction vector is directed along the cylinder axis, in other words, along the normal to $S$.

The intensity of cyclotron radiation has an angular distribution, and along the direction of the magnetic field its intensity is two times greater than in the perpendicular direction [5]. Thus, electromagnetic waves are emitted from the crystal both through the area of the ingot base $S$ and through the side surface with the height $\delta$. When the radius of the ingot base is much higher than $\delta$, then electromagnetic radiation through the side walls of the ingot can be neglected.

In accordance with (7), for the cyclotron radiation power of a semiconductor crystal, we have

$P_{S}=\frac{n e^{2} \omega_{B}^{2} v_{T}^{2}}{12 \pi \varepsilon_{0} \varepsilon c^{3}} S \delta$.

The thickness of the radiating layer $\delta$ is determined by the frequency $\omega_{B}$.

In the situation where $\omega_{B}<\omega_{P}, \omega_{P}$ is the plasma frequency of electrons equal [7]

$\omega_{P}=\left(\frac{n e^{2}}{\varepsilon_{0} \varepsilon m_{n}}\right)^{\frac{1}{2}}$,

and the condition $\omega_{B} \tau \gg>1$ is not fulfilled or, equivalently, $\mu B>>1$ (the latter relation follows from (1), taking into account that the relaxation time $\tau=m_{n} \mu_{n} / e$ [8], where $\mu_{n}$ is the mobility of electrons in the semiconductor, $\omega_{B} \tau=\mu B-$ number of revolutions that electron will complete before elastic scattering by ions and impurity atoms, dislocations, acoustic and optical phonons), the layer thickness $\delta$ coincides with the depth of the classical skin layer [9]

$\delta_{c}=c\left(\frac{2 \varepsilon_{0}}{\omega_{B} \sigma}\right)^{\frac{1}{2}}$,

where $\sigma$ is the conductivity of the semiconductor.

In this case, in accordance with the relations (1), (9) and (11), the power of cyclotron radiation of a semiconductor crystal is proportional to $\omega_{B}$, that is, $B$ to 
the power 3/2, and also to the square root of the concentration of free charge carriers.

When $\omega_{B}<\omega_{P}$ and $\omega_{B} \tau>>1(\mu B>>1)$, then $\delta$ is the thickness of the skin-layer of the plasma equal to [9]

$$
\delta_{P}=\frac{c}{\omega_{P}}
$$

and does not depend on the frequency of electromagnetic radiation.

Thus, the power of the cyclotron radiation, as follows from the relations (1), (9), (10) and (12), is proportional to $\omega_{B}^{2}$, hence to $B^{2}$, as well as in the previous case $\sqrt{n}$.

When $\omega_{B}>\omega_{P}$, solid-state plasma becomes transparent to electromagnetic radiation. However, in this case it is necessary to take into account its absorption by free charge carriers. The latter circumstance precisely determines the thickness of the layer $\delta_{n}$. As $\delta_{n}$, it is necessary to take the value inverse to the absorption coefficient $\delta_{n}=\alpha^{-1}$, the expression for which has the form [8]

$$
\alpha=\frac{\sigma}{c \bar{n} \varepsilon_{0}\left[1+\left(\frac{m_{n} \mu_{n} \omega}{e}\right)^{2}\right]},
$$

where $\omega$ is the circular frequency of electromagnetic radiation (in our case $\omega=\omega_{B}$ ), $\bar{n}$ is the real part of the complex refractive index, and here $\bar{n}^{2}=\varepsilon+\chi^{2}$, where $\chi=\alpha c / 2 \omega$ is the absorption index (extinction coefficient).

We note that the dependence $\alpha$ on $\omega^{-2}$, when the unity in the denominator of (13) can be neglected, is well satisfied, for example for InSb and Ge [8]. In the general case, it must be taken into account that the relaxation time and, consequently, the carrier mobility, are defined by the nature of the scattering centers and the exponent of the frequency dependence $\alpha$ for various semiconductor crystals can vary within rather wide limits [8].

Taking into account the relations (1), (13) and considering the case when $\varepsilon>>\chi^{2}$, that is, $\bar{n}=\sqrt{\varepsilon}$, we get

$\delta_{n}=\frac{c \varepsilon_{0} \varepsilon^{1 / 2}\left[1+\left(\mu_{n} B\right)^{2}\right]}{\sigma}$,

while $\left(\mu_{n} B\right)^{2}>>1$

$\delta_{n}=\frac{c \varepsilon_{0} \varepsilon^{1 / 2}\left(\mu_{n} B\right)^{2}}{\sigma}$.

Substituting (14) into (9), taking into account (1) and replacing $\sigma$ by $e n \mu_{n}$, we obtain

$P_{S}=\frac{e^{3} v_{T}^{2} B^{2}\left[1+\left(\mu_{n} B\right)^{2}\right]}{12 \pi \varepsilon^{1 / 2} c^{2} \mu_{n} m_{n}^{2}} S$,

while $\left(\mu_{n} B\right)^{2}>>1$
$P_{S}=\frac{e^{3} v_{T}^{2} \mu_{n} B^{4}}{12 \pi \varepsilon^{1 / 2} c^{2} m_{n}^{2}} S$.

As it follows from (16) and (17), the radiation power of electromagnetic waves emitted by a semiconductor crystal does not depend on the concentration of charge carriers and increases with the frequency, that is, with an increase of $B$ in the power law. At the same time, with increasing $\omega_{B}\left(\omega_{B} \leq \omega_{B \max }\right)$ in certain frequency ranges, cyclotron radiation is suppressed due to lattice absorption by characteristic phonons and their combinations, as well as electron transitions between individual subbands [8]. This circumstance reduces the degree of deviation of the plasma of the radiating layer from the state of thermodynamic equilibrium.

In conclusion we note, since electromagnetic radiation is emitted from a semiconductor crystal with a dielectric permittivity $\varepsilon$ into air with a dielectric constant equal to unity, a partial reflection of electromagnetic waves occurs at the semiconductor-air interface. So, with a normal incidence of the electromagnetic wave on the interface, the reflection coefficient has the form [10]:

$R=\left[\left(\varepsilon^{1 / 2}-1\right) /\left(\varepsilon^{1 / 2}+1\right)\right]^{2}$.

\section{Comparison with experiment}

In the experiments [4] on the investigation of cyclotron radiation of semiconductor crystals, a permanent magnet with an induction at the center of the air gap $B=0.45 \mathrm{~T}$ and a cylindrical ingot $p-\mathrm{Ge}$ with its height $4.6 \cdot 10^{-2} \mathrm{~m}$, base diameter $2.9 \cdot 10^{-2} \mathrm{~m}$, specific electrical resistance $0.44 \mathrm{Ohm} \cdot \mathrm{m}\left(p=6.1 \cdot 10^{19} \mathrm{~m}^{3}\right)$ were used.

We will estimate the parameters of cyclotron radiation. The cyclotron frequency of light holes (for which $m_{p}=0.043 m_{0}$, where $m_{0}$ is the mass of free electron) was $2.9 \cdot 10^{11} \mathrm{~Hz}$ in accordance with the relation (1). Taking into account (10) and $\varepsilon=16.2$, the plasma frequency $v_{P}=0.84 \cdot 10^{11} \mathrm{~Hz}$.

Since $\quad v_{B}>v_{p} \quad$ and $\quad \mu_{B} B=0.085<<1$ $\left(\mu_{B}=0.19 \mathrm{~m}^{2} \mathrm{~V}^{-1} \mathrm{~s}^{-1}\right)$, to calculate the cyclotron radiation power of the ingot, it is necessary to use the formula (16). Then, we have $P_{S}=44 \mu \mathrm{W}$. Note that the real power of the cyclotron radiation of a semiconductor crystal will be less than the calculated value due to the reflection of electromagnetic waves from the ingot surface. At normal incidence of the wave, for germanium the reflection factor $R=0.362$.

The results of heating liquid of $40 \mu \mathrm{l}$ volume by cyclotron radiation of a germanium ingot are presented in [4]. Being based on the well-known formula for the heat transferred to the body $Q=P_{S} t=c m \Delta T$, where $\Delta T$ is the change in temperature when heating, $c$ - specific heat of water, $t$ - heating time, and $m$ - mass of the liquid, as well as taking into account that the maximum temperature change $\Delta T$ is reached at the 16-th minute and is $0.09{ }^{\circ} \mathrm{C}$, we obtain that the radiation power is of 
the order of $15 \mu \mathrm{W}$. This value is less than the power of the cyclotron radiation of a germanium ingot that is $44(1-0.362) \mu \mathrm{W}=28 \mu \mathrm{W}$. The difference in power values is obviously a consequence of a number of operating factors. Basically, it is caused by the partial reflection of electromagnetic waves from the surface of the liquid, the heat transfer of the latter to the surrounding medium, losses in the system and, as it was shown in [4], a competing process of cooling water. The angular distribution of cyclotron radiation caused by the difference in the $R$ values for the waves incident at an angle to the semiconductor surface contributes.

\section{Conclusion}

Analytic expressions for calculating the power of cyclotron radiation of semiconductor crystals in situations when the thickness of the radiating layer coincide with that of the classical skin layer, the skin layer of the plasma, and the absorption layer by free charge carriers have been obtained. In all cases, the power of radiation depends on the induction of magnetic field in accordance with the power law. At the same time, the exponent has been varied within fairly wide limits. This fact agrees with the results of [2], where the quadratic dependence of power corresponding to the terahertz pulse from InAs on the magnetic induction was observed under irradiation of the crystal surface with femtosecond laser pulses. The theoretical calculations of the radiation power are in satisfactory agreement with the experimental results presented in [4].

\section{References}

1. Klimenko O.A., Mityagin Yu.A., Savinov S.A., Murzin V.N., Dyakonova N.V., Solignac P. and Knap W. Terahertz wide range tunable cyclotron resonance $p$-Ge laser. J. Phys. Conf. 2009. 193, No 1. P. 012064-1-012064-4.

2. Ohtake H., Murakami H., Yano T., Ono Sh., Sarukura N., Tarahashi H., Suzuki Y., Nishijima G., Watanabe K. Anomalous power and spectrum dependence of terahertz radiation from femtosecond-laser-irradiated indium arsenide in high magnetic fields up to $14 \mathrm{~T}$. Appl. Phys. Lett. 2003. 82, No 8. P. 1164-1166.

3. Klimenko O.A., Mityagin Yu.A., Videlier H., Teppe F., Dyakonova N.V., Consejo C., Bollaert S.,
Murzin V.N., and Knap W. Terahertz response of InGaAs field effect transistors in quantizing magnetic fields. Appl. Phys. Lett. 2010. 97, No 2. P. 0022111-1-0022111-3.

4. Milenin G.V., Milenin V.V., Red'ko R.A. Highfrequency electromagnetic radiation of germanium crystals in magnetic fields. Semiconductor Physics, Quantum Electronics and Optoelectronics. 2017. 20, No 2. P. 231-234.

5. Bekefi J. Radiation Processes in Plasma. Wiley, New York, 1966.

6. Wenzel E.S. Probability Theory. Moscow, Vysshaya shkola, 2001 (in Russian).

7. Smith R.A. Semiconductors, 2nd ed. Cambridge University Press, London, 1979.

8. Shalimova K.V. Physics of Semiconductors. St-Petersburg: Publ. House "Lan", 2010 (in Russian).

9. Lifshitz E.M., Pitaevsky L.P. Physical Kinetics, in: Landau L.D., Lifshitz E.M. Theoretical Physics. vol. X. Moscow, Nauka, 1979 (in Russian).

10. Vlasov A.A. Macroscopic Electrodynamics. Moscow, Fizmatlit, 2005 (in Russian).

\section{Authors and CV}

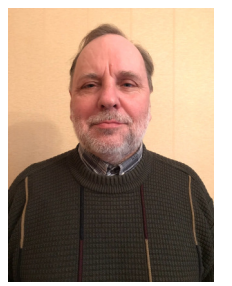

Milenin G.V.: PhD "Solid-State Physics", Senior Researcher.

V. Lashkaryov Institute of Semiconductor Physics, National Academy of Sciences of Ukraine E-mail:milenin.gv@gmail.com

Milenin V.V.: $\quad \mathrm{PhD} \quad$ "Solid-State Physics", Senior Researcher

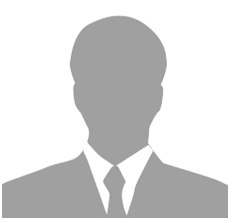

$V$. Lashkaryov Institute of Semiconductor Physics, National Academy of Sciences of Ukraine E-mail:

milenin.viktor.vlad@gmail.com

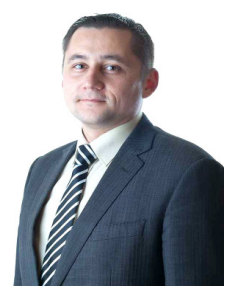

Red'ko R.A.: $\mathrm{PhD}$ "Solid-State Physics", Senior Researcher, Associate Professor.

V. Lashkaryov Institute of Semiconductor Physics, National Academy of Sciences of Ukraine E-mail: redko.rom@gmail.com 